\title{
Abnormal Phase Coupling in Parkinson's Disease and Normalization Effects of Subthreshold Vestibular Stimulation
}

\author{
Soojin Lee ${ }^{1,2}$, Aiping Liu' ${ }^{2,3 *}$, Z. Jane Wang ${ }^{1,4}$ and Martin J. McKeown ${ }^{2,5}$ \\ ${ }^{1}$ School of Biomedical Engineering, University of British Columbia, Vancouver, BC, Canada, ${ }^{2}$ Pacific Parkinson's Research \\ Centre, Vancouver, BC, Canada, ${ }^{3}$ Department of Electronic Science and Technology, University of Science and Technology \\ of China, Hefei, China, ${ }^{4}$ Department of Electrical and Computer Engineering, University of British Columbia, Vancouver, BC, \\ Canada, ${ }^{5}$ Department of Medicine (Neurology), University of British Columbia, Vancouver, BC, Canada
}

\section{OPEN ACCESS}

Edited by:

Matt J. N. Brown,

California State University, Sacramento, United States

Reviewed by:

Görsev Yener,

Dokuz Eylül University, Turkey Antonio Ivano Triggiani,

University of Foggia, Italy

*Correspondence:

Aiping Liu

aiping/@ece.ubc.ca

Received: 22 November 2018 Accepted: 19 March 2019 Published: 03 April 2019

Citation:

Lee S, Liu A, Wang ZJ and McKeown MJ (2019) Abnormal Phase

Coupling in Parkinson's Disease and Normalization Effects of Subthreshold Vestibular

Stimulation

Front. Hum. Neurosci. 13:118. doi: 10.3389/fnhum.2019.00118
The human brain is a highly dynamic structure requiring dynamic coordination between different neural systems to perform numerous cognitive and behavioral tasks. Emerging perspectives on basal ganglia (BG) and thalamic functions have highlighted their role in facilitating and mediating information transmission among cortical regions. Thus, changes in BG and thalamic structures can induce aberrant modulation of corticocortical interactions. Recent work in deep brain stimulation (DBS) has demonstrated that externally applied electrical current to BG structures can have multiple downstream effects in large-scale brain networks. In this work, we identified EEG-based altered resting-state cortical functional connectivity in Parkinson's disease (PD) and examined effects of dopaminergic medication and electrical vestibular stimulation (EVS), a noninvasive brain stimulation (NIBS) technique capable of stimulating the BG and thalamus through vestibular pathways. Resting EEG was collected from 16 PD subjects and 18 age-matched, healthy controls $(\mathrm{HC})$ in four conditions: sham (no stimulation), EVS1 (4$8 \mathrm{~Hz}$ multisine), EVS2 (50-100 Hz multisine) and EVS3 (100-150 Hz multisine). The mean, variability, and entropy were extracted from time-varying phase locking value (PLV), a non-linear measure of pairwise functional connectivity, to probe abnormal cortical couplings in the PD subjects. We found the mean PLV of Cz and C3 electrodes were important for discrimination between PD and $\mathrm{HC}$ subjects. In addition, the PD subjects exhibited lower variability and entropy of PLV (mostly in theta and alpha bands) compared to the controls, which were correlated with their clinical characteristics. While levodopa medication was effective in normalizing the mean PLV only, all EVS stimuli normalized the mean, variability and entropy of PLV in the PD subject, with the exact extent and duration of improvement a function of stimulus type. These findings provide evidence demonstrating both low- and high-frequency EVS exert widespread influences on cortico-cortical connectivity, likely via subcortical activation. The improvement observed in PD in a stimulus-dependent manner suggests that EVS with optimized parameters may provide a new non-invasive means for neuromodulation of functional brain networks.

Keywords: Parkinson's disease, electrical vestibular stimulation, EEG, phase locking value, cortical oscillations, sample entropy, sparse discriminant analysis 


\section{INTRODUCTION}

Parkinson's disease (PD), the second most common neurodegenerative disease (Scandalis et al., 2001), is characterized by motor symptoms such as bradykinesia, tremor, rigidity and impaired balance and gait as well as nonmotor complications, resulting primarily from degeneration of dopaminergic neurons in the substantia nigra pars compacta (SNc) (Davie, 2008). Several electrophysiology studies using local field potential (LFP) recordings demonstrated that, in the dopamine-deficient state, the neuronal synchronization in the basal ganglia (BG) is exaggerated at frequencies in the beta range $(13-30 \mathrm{~Hz})$ (Brown and Williams, 2005; Eusebio et al., 2009; Litvak et al., 2012; Oswal et al., 2013). These beta oscillations are also highly synchronized with sensorimotor areas (Brown et al., 2001; Marsden et al., 2001; Cassidy et al., 2002; Williams et al., 2002) as well as muscle activity of upper limbs during movement (Marsden et al., 2001). This excessive beta synchronization is considered to be, in part, responsible for the Parkinsonian symptoms and thus reducing the abnormal synchronization with deep brain stimulation (DBS) has shown to be an effective therapy.

Recent fMRI findings have highlighted that large-scale cortical resting-state functional connectivity ( $\mathrm{rsFC}$ ) is altered in $\mathrm{PD}$, possibly as a result of BG impairment effects on cortical-BG networks (Helmich et al., 2010). The striatum, a subcortical region significantly affected with dopamine depletion in PD, has altered FC with inferior parietal, temporal, and motor cortices (Helmich et al., 2010), which supports that PD-induced connectivity changes can be seen beyond local subcortical regions. In addition to effects on BG-cortical FC, impairment in the BG can also alter cortico-cortical connectivity. Diminished interhemispheric connectivity in sensorimotor cortical regions (Seibert et al., 2012) and reduced rsFC in widespread regions including inferior frontal, superior parietal, and occipital regions (Dubbelink et al., 2014) have been shown to be implicated with disease duration and cognitive dysfunctions in PD.

Inferring pathological cortico-cortical connectivity in $\mathrm{PD}$ solely based on evidence from fMRI alone may not provide a complete picture, as fMRI has limited temporal resolution. Electrophysiology can provide complementary information as it measures spontaneous synchronous activity of a large population of neurons occurring on a millisecond time scale. A simultaneous LFP-electroencephalography (EEG) study reported that the dynamics of LFP synchrony in STN is related to the dynamics of cortical synchrony (Ahn et al., 2015), and BG DBS modulates cortical phase coupling measured with EEG (Silberstein et al., 2005; Smolders et al., 2013).

One of the most widely-used method to quantify the couplings between oscillatory signals recorded at pairs of electrodes placed on the scalp in EEG is to look at their phase relationships (Klimesch et al., 2008; Fell and Axmacher, 2011). If cortical activities at two different regions are coupled, their phase angle differences tend to be consistent across time. Phase locking value (PLV) quantifies the strength of the phase coupling between two oscillatory signals, bounded between zero and one indicating a completely random and perfectly coupled relationship, respectively. Interregional phase synchronization has been shown to reflect specific neural activity coding different cognitive functions (Klimesch et al., 2001; Hanslmayr et al., 2005), motor behaviors (Andres and Gerloff, 1999; for a review, see Sauseng and Klimesch, 2008) and pathological brain states (Spencer et al., 2004; Sakkalis et al., 2007; Vakorin et al., 2016). However, to date, only a few studies have examined phase-based rsFC across broad cortical regions and different frequency bands in PD (Silberstein et al., 2005; Moazami-Goudarzi et al., 2008; George et al., 2013; He et al., 2017).

Most of the EEG connectivity studies to date have employed magnitude squared coherence. PD subjects exhibit excessive EEG coherence (Silberstein et al., 2005; George et al., 2013), especially in the beta band, in the off-medication condition that is decreased by medication (George et al., 2013). For PD subjects on-medication, enhanced coherence in the frontal regions in the theta $(4-6 \mathrm{~Hz})$, beta $(12-18 \mathrm{~Hz})$, and gamma $(30-45 \mathrm{~Hz})$ (Moazami-Goudarzi et al., 2008) and altered interhemispheric beta coherences in the midtemporal and frontal areas (He et al., 2017) can be observed, indicating the multifarious role of dopamine in the control of oscillatory activity, in and beyond the BG. However, coherence is different from PLV in that it relies on the assumption of linearity and stationarity in the signals and is calculated independently for each frequency, which is then scaled by the amplitudes of the signals. PLV-based connectivity, which do not rely on the strict assumptions underlying coherence, might be more suitable for non-linear and non-stationary dynamics of neural oscillations, and sheds a new light on pathophysiological brain networks as it has not been explored yet in PD.

Recent progress in non-invasive brain stimulation (NIBS) has demonstrated its capability to modulate cortical oscillations (Helfrich et al., 2014; Vossen et al., 2015; Amengual et al., 2017) and interregional couplings, indicating its potential applications as an effective therapeutic technique for PD. Electrical vestibular stimulation (EVS) is a NIBS technique that delivers weak current to the mastoid processes and modulates firing rates of vestibular afferents, which then activates various cortical and subcortical regions including the BG and thalamus (Bense et al., 2001; Utz et al., 2010; Lopez et al., 2012). Similar to transcranial electrical stimulation (tES), EVS stimuli can take the form of direct current (DC), alternating current (AC), or random noise (RN) and stimulation effects vary according to stimulus types. While DCEVS perturbs perception of orientation and locomotion and has been widely utilized in postural balance control research (St George and Fitzpatrick, 2011), RN-EVS has demonstrated its efficacy in motor functions (Yamamoto et al., 2005; Pan et al., 2008; Lee et al., 2015) and modulation of EEG oscillatory rhythms across broad cortical regions in PD (Kim et al., 2013). It is conceivable, therefore, that EVS may be able to modulate cortical couplings, which has not been explored yet.

To establish the potential of EVS as a therapeutic intervention to modulate abnormal cortical couplings in PD, we investigated whether resting-state cortical couplings, as measured as PLV, were altered in unmedicated PD subjects, and determined if EVS had any normalizing effects. Specifically, we applied three novel EVS stimuli, each restricted to a specific frequency band, to PD and healthy subjects and examined how the different stimuli 
affected both the strength and temporal variation of aberrant couplings in PD.

\section{MATERIALS AND METHODS}

\section{Participants}

Twenty PD patients and 22 age- and gender-matched healthy controls (HC) participated in this study. Patients with atypical parkinsonism or other neurological disorders were excluded from the study, and all included PD patients were classified as having mild to moderate stage PD (Hoehn and Yahr Stage 1-2). Four $\mathrm{PD}$ and four HC subjects were excluded in the data analysis due to severe muscle artifacts in their EEG recordings. Therefore, $16 \mathrm{PD}$ (7 males; age: $67.3 \pm 6.5$ years) and $18 \mathrm{HC}$ (9 males; age: $67.6 \pm 8.9$ years) subjects were included in the analysis (Table 1). All subjects did not have any reported vestibular or auditory disorders and were right-handed. The study protocol was approved by the Clinical Research Ethics Board at the University of British Columbia (UBC) and the recruitment was conducted at the Pacific Parkinson's Research Centre (PPRC) in UBC. All subjects gave a written informed consent prior to participation.

\section{Study Protocol}

As individuals have inherently subjective perception of EVS, we utilized systematic procedures that have been previously used in determining subliminal current level (Lee et al., 2015). The measured individual threshold level was in the range of $0.23-$ $1.1 \mathrm{~mA}$. After the threshold was determined, the subjects were comfortably seated in front of a computer screen and were instructed to focus their gaze on a continuously displayed fixed target while EEG was being recorded. EEG was first recorded without stimulation for $20 \mathrm{~s}$ and EVS were then delivered for a fixed duration of $60 \mathrm{~s}$, followed by an EVS-off period for $20 \mathrm{~s}$ (post-stimulation). During the stimulation period, EVS was applied at $90 \%$ of the individual threshold level.

EEG was recorded from the subjects in 4 different conditions: Sham (no stimulation), EVS1, EVS2 and EVS3 (for details, see

TABLE 1 | Demographic and clinical characteristics of the patients with Parkinson's disease $(\mathrm{PD})$ and healthy controls $(\mathrm{HC})$.

\begin{tabular}{lcc}
\hline & PD & HC \\
\hline Age (years), mean (SD) & $67.3(6.5)$ & $67.6(8.9)$ \\
Gender, n (male/female) & $7 / 9$ & $9 / 9$ \\
Disease duration (years), mean & $7.4(4.3)$ & - \\
(SD) & & - \\
aUPDRS II, mean (SD) & $14.8(8.1)$ & - \\
bUPDRS III, mean (SD) & $22.1(8.9)$ & - \\
Hoehn and Yahr scale, mean & $1.3(1-2)$ & - \\
(range) & & \\
Levodopa equivalent daily dose & $635.9(356.4)$ & \\
(mg), mean (SD) (Tomlinson & & \\
et al., 2010) & & \\
\hline
\end{tabular}

${ }^{a}$ Motor aspects of experiences of daily living. ${ }^{b}$ Motor symptoms. section EVS). EEG recording was first performed in the sham condition and the EVS conditions were randomly ordered. We allowed a 2 min break between each condition to prevent any potential post-stimulation effects carried over from the previous EVS conditions.

The HC subjects performed the protocol once, whereas PD subjects performed it twice in off-medication (PDMOFF) and on-medication (PDMON) conditions on the same day. The PD subjects stopped taking their normal L-dopa medication at least $12 \mathrm{~h}$, and any dopamine agonists $18 \mathrm{~h}$ prior to the EEG recording. United Parkinson's Disease Rating Scale (UPDRS) Parts II and III were assessed in the off-medication condition. Immediately after finishing the EEG acquisition, they took their regular dose of L-dopa medication and rested for $1 \mathrm{~h}$. After the break, EEG was recorded in the on-medication condition.

\section{EVS}

EVS was delivered through pre-gelled $\mathrm{Ag} / \mathrm{AgCl}$ electrodes (BIOPAC Systems Inc., CA, United States) placed in bilateral, bipolar fashion over the mastoid process behind each ear. Nuprep $^{\mathrm{TM}}$ skin prep gel was used to clean skin for better electrode contact and to reduce resistance during stimulation. Stimulation waveforms were generated on a computer using MATLAB (R2018a, MathWorks, MA, United States) and converted to an analog signal using a NI USB-6221 BNC digital acquisition module (National Instruments, TX, United States). The analog voltage signals were then passed to a constant current stimulator (DS5, Digitimer, United Kingdom), which was connected to the stimulating electrodes.

Three multisine signals in different frequency bands (EVS1: 4-8 Hz; EVS2: 50-100 Hz; EVS3: 100-150 Hz) were used (Figure 1A). Multisine signals are designed to concentrate power at a precise number of frequencies within the bandwidth of interest, which is advantageous compared to other excitation signals (e.g., a white noise or swept sine) as there is no spectral leakage. Each multisine signals were designed to have the frequencies of sinusoids $\left(f_{i}\right)$ uniformly distributed every $0.2 \mathrm{~Hz}$ and the phases $\left(\phi_{i}\right)$ chosen to minimize the crest factor using a clipping algorithm (Van der Ouderaa et al., 1988) in order to generate a flat amplitude of the signal and thus improve subject's comfort:

$$
x(t, \phi)=a \cdot \sum_{i=1}^{n} \cos \left(2 \pi f_{i} t+\phi_{i}\right)
$$

where $x(t, \phi)$ is the multisine, $a$ is the amplitude, $f_{i}$ and $\phi_{i}$ are the frequency and phase, and $i$ is the index of each sinusoidal component (e.g., $f_{1}, f_{2}, \ldots, f_{n}=4.0,4.2, \ldots, 8.0 \mathrm{~Hz}$ for EVS1).

\section{EEG Recording}

Data were recorded from 27 scalp electrodes using a 64-channel EEG cap (Neuroscan, VA, United States) and a Neuroscan SynAmps2 acquisition system (Neuroscan, VA, United States) at a sampling rate of $1 \mathrm{kHz}$. Recording electrodes were positioned according to the International 10-20 placement standard with one ground and one reference electrode located between $\mathrm{Cz}$ and $\mathrm{CPz}$ (Figure 1B). Impedances were kept below $15 \mathrm{k} \Omega$ using 

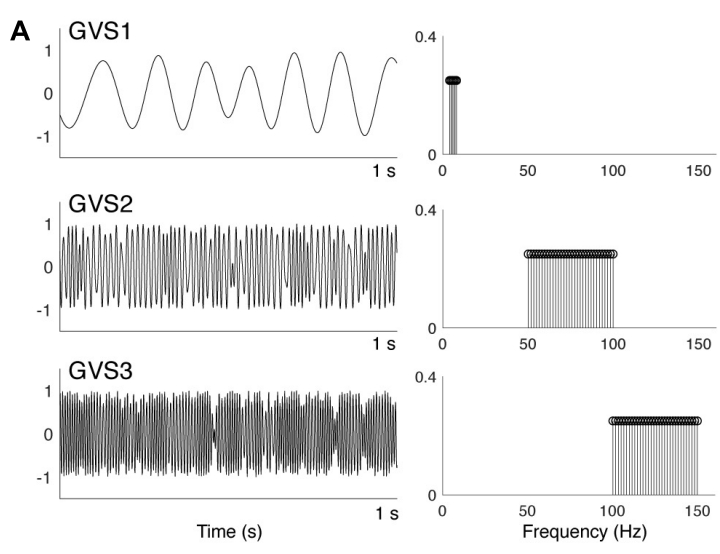

B

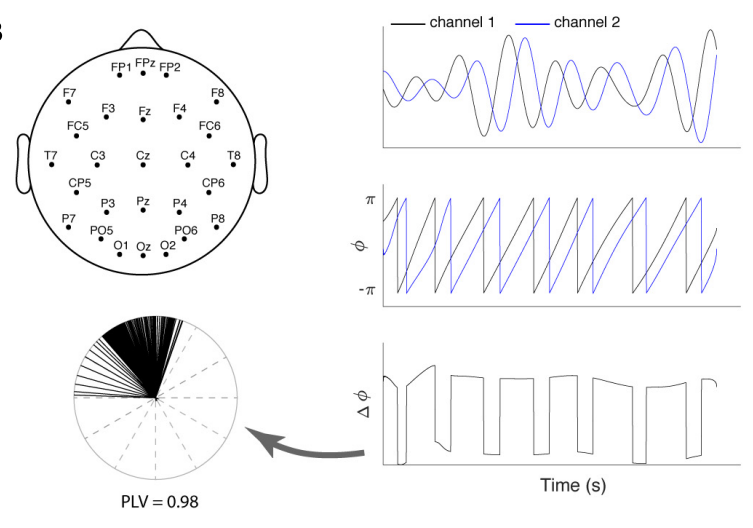

C
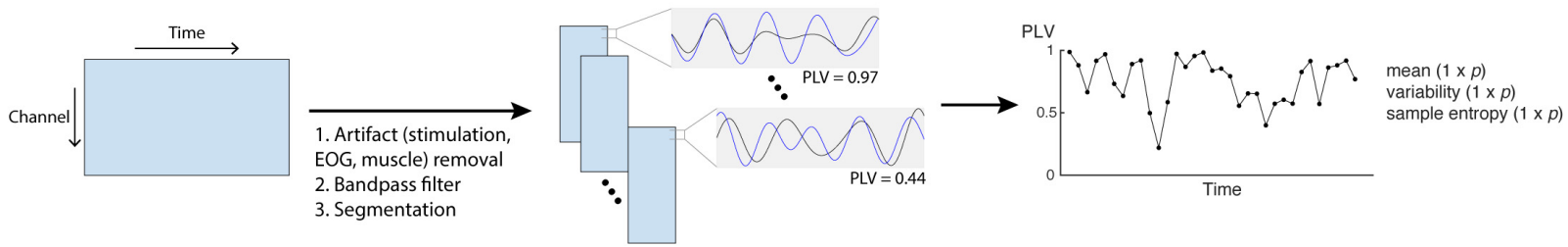

FIGURE 1 | (A) Time and frequency plots of the three types of multisine stimulus given at 90\% individual threshold level (EVS1: 4-8 Hz; EVS2: 50-100 Hz; EVS3: 100-150 Hz). (B) Placement of 27 EEG electrodes and PLV calculation. Hilbert transform is applied to the two signals to extract the instantaneous phases. The phase differences calculated at each time point are represented into unit vectors in the complex plane and PLV is computed to evaluate the spread of the distribution (Lachaux et al., 1999; Mormann et al., 2000). (C) The procedure to extract phase locking value (PLV) time series. For each subject, preprocessing steps were first applied to the raw EEG data in order to remove high-voltage stimulation artifacts as well as cardinal artifacts caused by eye movements [electrooculography (EOG)] or muscle movement. The cleaned data were bandpass filtered into four different frequency bands (theta: 4-8 Hz; alpha: 8-13 Hz; beta: 13-30 Hz; gamma: $30-45 \mathrm{~Hz}$ ) and segmented into epochs. PLV between a pair of electrodes in each epoch was computed to generate the time series, and its mean, variability, and sample entropy were calculated. Each subject has a $1 \times p$ vector for the mean, variability and sample entropy $(p=1,404=351$ pairs $\times 4$ frequency bands).

Electro-Gel (Electrode-Cap International, OH, United States). No clipping of EEG was observed during stimulation.

\section{EEG Preprocessing}

The EEG data were bandpass filtered between 3 and $45 \mathrm{~Hz}$ using a two-way finite impulse response (FIR) filter (the "eegfilt" function in EEGLAB). High-voltage stimulation artifacts during EVS2 and EVS3 were removed using the digital filters. The artifacts during EVS1 were removed using a quadrature-IVA method (Lee et al., 2019). Data were then re-referenced to the average reference (linked earlobe) and ocular artifacts (EOG) were corrected based on cross-correlation with the reference EOG channels using the AAR toolbox included in EEGLAB. The cleaned EEG data were bandpass filtered into four conventional EEG frequency bands (Groppe et al., 2013): theta $(4-8 \mathrm{~Hz})$, alpha $(8-13 \mathrm{~Hz})$, beta $(13-30 \mathrm{~Hz})$, and gamma $(30-45 \mathrm{~Hz})$. The bandpass-filtered data were then segmented into non-overlapping epochs. Epoch sizes were determined such that the epochs include around four cycles at a center frequency of the selected bandwidth (Martin and Schröder, 2016), resulting in epoch sizes of $600,400,200$, and $100 \mathrm{~ms}$ for the theta, alpha, beta, and gamma bands.

\section{Phase Locking Value (PLV)}

PLV evaluates the spread of the distribution of phase angle differences between pairs of electrodes over time
(Lachaux et al., 1999; Mormann et al., 2000; Figure 1B). The connectivity is measured from this spread such that strongly clustered phase differences between two electrodes result in the PLV value close to one, indicating a strong connectivity between the signals. If there is no phase dependence, PLV value becomes zero.

To calculate the PLV, instantaneous phase angles were obtained by applying the Hilbert transformation to the bandpassfiltered data. Then, the PLV between two signals A and B was computed as Bruña et al. (2018):

$$
P L V_{A, B}=\frac{1}{T}\left|\sum_{t=1}^{T} e^{-i\left(\varphi_{A}(t)-\varphi_{B}(t)\right)}\right|
$$

where $T$ is the number of time points and $\varphi(t)$ is the instantaneous phase angles of each EEG signal. The PLV was computed for each epoch, resulting in times series of the PLV computed from all pairs of 27 electrodes and the four frequency bands (1,404 time series in total). Three temporal features were extracted from each PLV time series for further analysis: the mean, variability (standard deviation), and sample entropy. Sample entropy is a non-linear measure to quantify the degree of complexity in a time series (Richman and Moorman, 2000), and has been applied to EEG data for clinical application such as classification (Bruce et al., 2009; Kumar et al., 2012) and epilepsy 
A

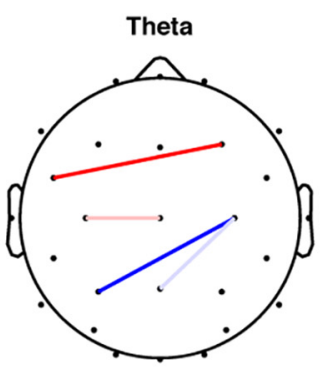

B

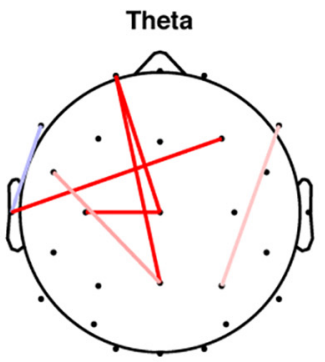

C

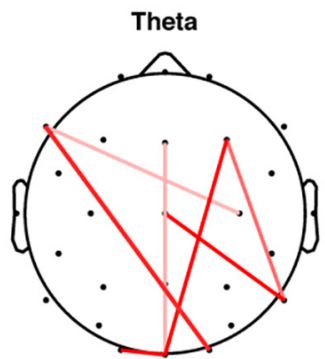

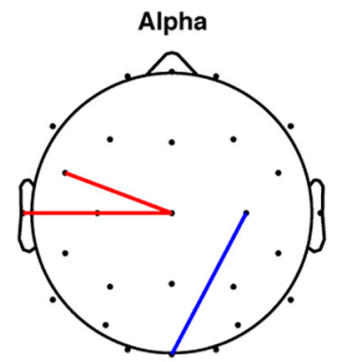
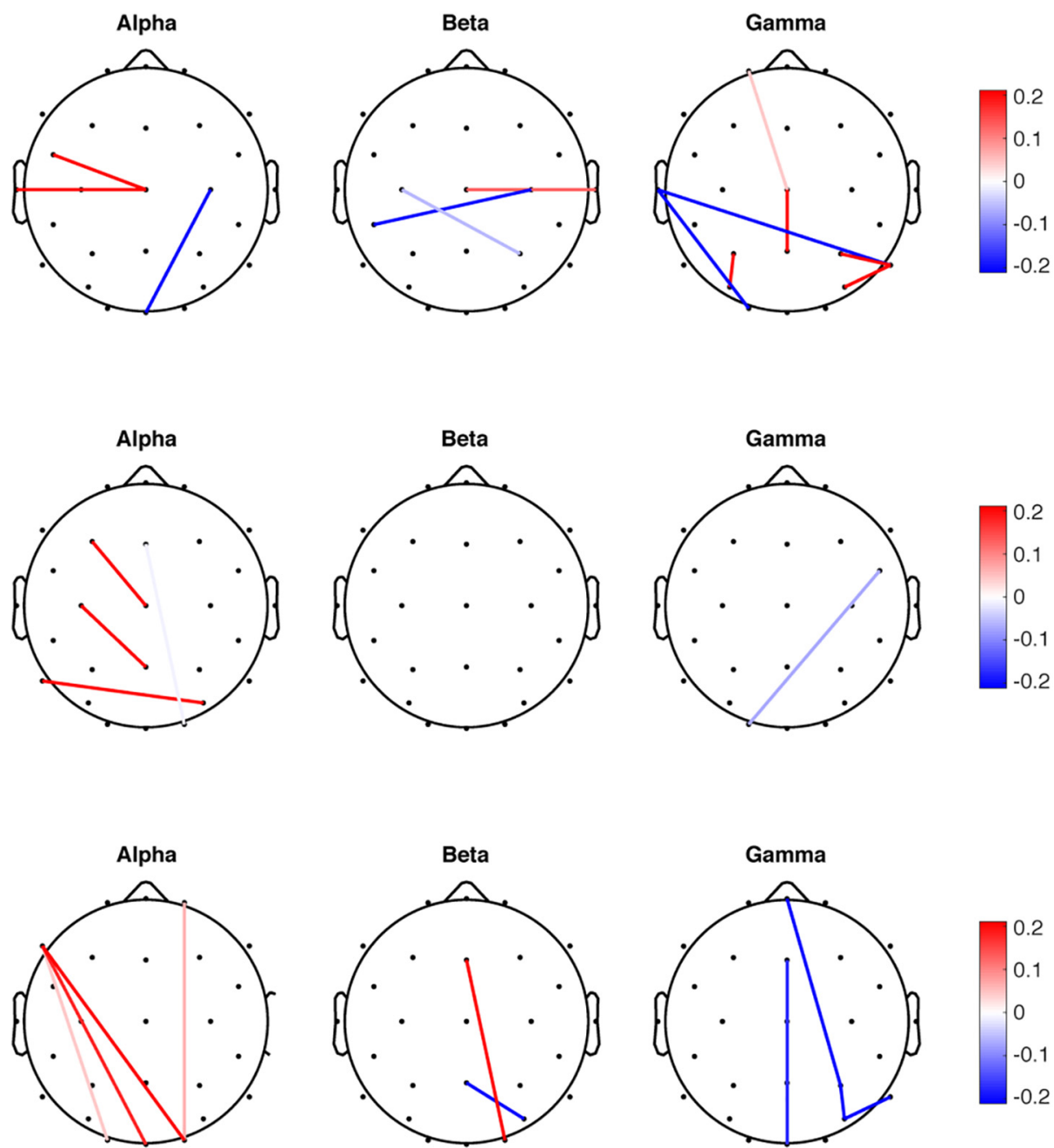

FIGURE 2 | Non-zero features selected by sparse discriminant analysis (SDA). SDA was applied to the mean, variability and entropy data sets, respectively, to discriminate the PDMOFF and HC groups. The non-zero weights in the sparse discriminant vectors are presented in the scalp maps. (A) Weights for the 17 selected features from the mean PLV data set. (B) Weights for the 12 selected features from the PLV variability data set. (C) Weights for the 17 selected features from the PLV entropy data set.

detection (Song et al., 2012). Tolerance $(r)$ and window length ( $m$ ) were specified to be 0.3 and 2, respectively, to compute the sample entropy based on Lake et al. (2002) and characteristics of our data sets.

\section{Sparse Discriminant Analysis}

Linear discriminant analysis (LDA) is a classical supervised classification technique that finds the most discriminative projections of a $N \times p$ data in a $p$-dimensional space such that the data projected into the low-dimensional subspace can be well partitioned into $k$ classes (Mai, 2013). In biomedical research, it has become an increasingly important topic to perform a classification with a high-dimensional data where the number of variables far exceeds the number of samples. In such highdimensional settings, LDA cannot be applied directly because of singularity of the sample covariance matrix. To overcome this limitation, various regularized versions of LDA have been proposed (Safo and Long, 2018). Sparse discriminant analysis (SDA) was proposed by Clemmensen et al. (2011) where an elastic net penalty and optimal scoring framework are applied to a highdimensional data to generate a sparse discriminant vector. The authors demonstrated that SDA outperforms other regularized methods such as shrunken centroids regularized discriminant analysis and sparse partial least squares regression. The details of the algorithm can be found in Clemmensen et al. (2011).

Here, we aim to classify the PDMOFF and HC groups in the baseline resting state (i.e., the sham condition) using the PLV features obtained above. The three data sets (mean, variability and sample entropy) have the same high-dimensional settings as each data set has the number of variables $(p=1,404)$ much greater than the number of samples (i.e., subjects). Therefore, we applied SDA to each data set to infer from the sparse discriminant vectors which combination of the electrode pairs and frequency bands are the most important features for the classification of 
the two groups. As in Clemmensen et al. (2011), we created the training set consisted of 26 subjects (12 PDMOFF and 14 $\mathrm{HC}$ ) and the test set of eight subjects (4 PDMOFF and $4 \mathrm{HC}$ subjects) and the tuning parameters for SDA (i.e., $\lambda$ and $\gamma$ for regularization penalties) were chosen using leave-one-out crossvalidation (LOOCV) on the training data. The models with the selected parameters were evaluated on the test data.

In the subsequent analyses, we investigated effects of L-dopa medication on the PLV features by applying the sparse discriminant vectors obtained from the above SDA to the data sets of the PDMON group in the sham condition. In the same manner, effects of EVS on the PLV features were evaluated by applying the same sparse discriminant vectors to the data sets in the EVS conditions.

\section{Statistical Analysis}

A one-way ANOVA was performed to compare the PLV features between groups followed by post hoc Tukey's honestly significant difference (HSD) test for multiple comparison correction. To evaluate effects of EVS on the PLV features within a group, a repeated measures ( $\mathrm{rm})$ ANOVA, with stimulation condition (sham, EVS1, EVS2, and EVS3) as the within-subject factor, was performed followed by post hoc Tukey's HSD test for multiple comparison correction. The rm ANOVA analysis was performed for online and after-effect conditions, respectively.

\section{RESULTS}

\section{SDA Classification Results and Selected Features}

SDA was performed for the mean, variability, and entropy PLV data sets independently to discriminate the PDMOFF and $\mathrm{HC}$ groups. Since there are two classes in the data, only one discriminant vector was obtained from each SDA. For the mean PLV data set, LOOCV on the training data resulted in the selection of 17 non-zero features (1.2\%) out of total 1,404 features (Figure 2A). There were both negative and positive weights for the selected features in each frequency band. Since the transformed PLV mean was greater for the PDMOFF (Figure 3A) than the HC group, the positive weights were interpreted as cortical couplings exaggerated in the PDMOFF group. 35\% of the selected features were associated with $\mathrm{Cz}$ over a broad frequency bandwidth, and the PDMOFF group had a stronger coupling strength for the features. In contrast, the features related to C4 had negative weights, indicating that these couplings are attenuated in the PDMOFF group. In the gamma band, decreased long-distance connectivity in the left temporal region (T7-O1 and T7-P8) and increased short-distance connectivity in the parietal region (P3-PO5, $\mathrm{P} 8-\mathrm{P} 4$, and $\mathrm{P} 8-\mathrm{PO} 6)$ were found to be related to the PDMOFF group. The training and test classification accuracy (fraction of correctly classified) were both $100 \%$.

For the PLV variability data set, 12 non-zero features $(0.85 \%)$ were selected and the largest number of the selected features was found in the theta band (Figure 2B), followed by the alpha and gamma bands. Note that positive weights are associated with the lower connectivity variability of the PDMOFF group because the transformed variability is lower for the PDMOFF group (Figure 3A). Decreased variability in the PDMOFF group was mostly associated with the frontal electrodes in the theta band and with F3-Cz, C3-Pz, and P7-PO6 in the alpha band. The classification accuracy for the training and test data sets were 100 and $87.5 \%$, respectively.

The SDA on the PLV entropy data set selected 17 nonzero features (1.2\%) and most of them were long-distance connectivity. Note that positive weights are associated with the connectivity with lower entropy for the PDMOFF group. In the theta and alpha bands, the entropy of the selected features was lower whereas in the gamma band the entropy was higher for the PDMOFF group compared to the HC group. In the beta band, the PDMOFF group had a lower entropy for Fz-O2 and higher entropy for Pz-PO6 than the HC group. The training and test classification accuracy were 96 and $87.5 \%$, respectively.

\section{Group Comparison of Baseline PLV Features}

The SDA discriminant vectors were applied to the data sets obtained from the PDMON group, and the group means of the transformed data are compared in Figure 3A. Significant group differences were found for the PLV features [PLV mean: $F(2,47)=41.68, P<0.001$; PLV variability: $F(2,47)=23.46$, $P<0.001$; PLV entropy: $F(2,47)=60.59, P<0.001]$. The PLV mean for the PDMOFF group was significantly higher than the HC group $(P<0.001)$, which was decreased by L-dopa medication $(P<0.01)$. The PLV variability was significantly lower in the PDMOFF compared to the HC group $(P<0.001)$, and the lower variability was associated with higher UPDRS2 scores (i.e., more severe difficulties of daily motor activities) $(r=-0.56, P=0.025$; Figure 3B). The medication slightly improved the variability in the PD subjects but the changes did not reach statistical significance $(P=0.096)$. The entropy of the PDMOFF group was lower than the HC group $(P<0.001)$ and the lower entropy was related to a longer disease duration $(r=-0.51, P=0.038$; Figure 3B). The medication did not improve the PLV entropy $(P=0.41)$.

\section{Online- and After-Effects of EVS}

Next, EVS effects on the PLV features were investigated. Specifically, we examined whether the effects are dependent on the stimulus types and sustained even after the stimulation ceases. Figures $4 \mathrm{~A}-\mathrm{C}$ show changes in the PLV mean for each group induced by EVS1, EVS2, and EVS3, respectively. The PLV mean was significantly modulated during stimulation in PDMOFF $[F(3,45)=11.16, P<0.001]$ and $\operatorname{HC}[F(3,51)=3.81, P<0.05]$ groups. All stimuli decreased the PLV mean in the PDMOFF group compared to the sham condition (EVS1: $P<0.001$; EVS2: $P<0.01$; EVS3: $P<0.01$ ), making it closer to the HC group, and the effects lasted in the post-stimulation period. EVS1 decreased the mean PLV greater than the other two stimuli and there was no continuing decrease in the post-stimulation period whereas EVS3 decreased the mean PLV less than EVS1 during stimulation and the effect continued in the post-stimulation period. In contrast, we found the opposite EVS effects for the HC group where EVS 
A
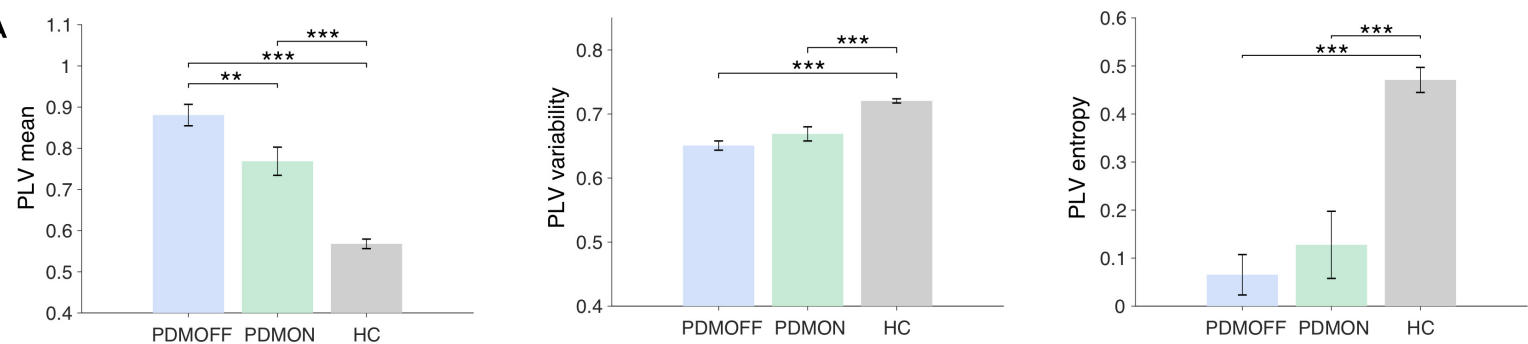

B
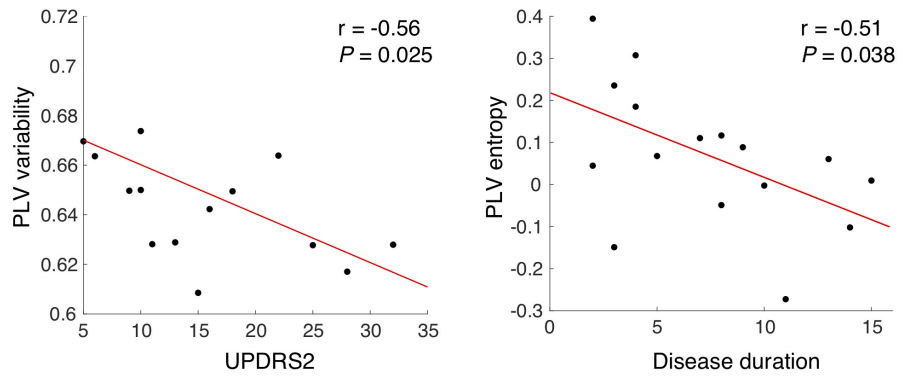

FIGURE 3 | (A) Group comparison of the discriminant component obtained from the SDA. The discriminant components were obtained by multiplying the discriminant vectors to the data sets from the sham condition. Bars and error bars indicate group means and s.e. Significant $P$-values from one-way ANOVA with post hoc Tukey's HSD test are indicated $\left({ }^{* *} P<0.01 ;{ }^{* * *} P<0.001\right)$. (B) Pearson correlations with clinical scores. The PLV variability and entropy of the PDMOFF subjects are significantly correlated with UPDRS2 and disease duration, respectively.

increased the PLV mean (EVS2: $P<0.05$; EVS3: $P<0.01)$. No significant effects of EVS were found in the PDMON group.

EVS effects on the PLV variability are presented in Figures 5A-C. There were significant online effects of stimulation on the PLV variability in $\operatorname{PDMOFF}[F(3,45)=4.43$, $P<0.01]$ and $\mathrm{HC}[F(3,51)=4.62, P<0.01]$ groups. EVS1 and EVS2 were found to have positive effects on the PDMOFF group, increasing the variability during stimulation (EVS1: $P<0.01$; EVS2: $P<0.05)$. Similar to the effects on the PLV mean, EVS1 induced the greatest increase in the variability during stimulation and the increased value tends to return to the baseline after the stimulation ceased whereas the effects of EVS2 and 3 were less during stimulation but lasted longer than that of EVS1. In the HC group, we found decreases in the PLV variability induced by EVS (EVS1: $P<0.01$; EVS2: $P<0.05$; EVS3: $P<0.05)$. EVS1 decreased the variability during the stimulation and the effect lasted in the post-stimulation period. EVS2 and EVS3 appeared to further decrease the variability in the post-stimulation period. For the PDMON group, all stimuli increased the PLV variability but the effects did not reach the statistical significance.

Figures 6A-C show EVS effects on the PLV entropy. The PLV entropy was significantly modulated during stimulation in $\operatorname{PDMOFF}[F(3,45)=4.65, P<0.01], \operatorname{PDMON}[F(3,45)=3.12$, $P<0.05]$, and $\mathrm{HC}[F(3,51)=4.25, P<0.01]$ groups. We found that all stimuli increased the entropy significantly in the PDMOFF group (EVS1: $P<0.01$; EVS2: $P<0.05$; EVS3: $P<0.05$ ), bring it closer to the HC group. The effects were greatest during stimulation and diminished in the poststimulation period, and EVS1 increased the largest amount of the entropy. For the PDMON group, EVS1 $(P<0.05)$ and
EVS2 $(P<0.05)$ increased the entropy significantly. While not statistically significant, increases in the entropy were also found during and post-EVS3 compared to the sham condition. The PLV entropy of the HC group changed in the opposite direction by EVS compared to the PD groups. Significant decreases in the entropy was observed with all stimuli [EVS1 $(P<0.01)$, EVS2 $(P<0.05)$ and EVS3 $(P<0.01)]$.

\section{DISCUSSION}

We investigated phase-based cortical connectivity in resting EEG in PD. To our knowledge, this is the first study that examined connectivity dynamics in PD by characterizing temporally fluctuating cortico-cortical couplings over broad frequency bands. The results from the current study on the time-varying connectivity provide novel insights into altered cortical dynamics derived from pathological BG changes in $\mathrm{PD}$.

\section{Disrupted Cortical Coupling Strength in the Motor Regions}

We found most changes in cortical coupling strength associated with PD (Figure 2A; 11 out of 17) were in key motor and parietal regions, including over the primary motor cortex (M1), supplementary motor area (SMA), premotor area (PMA), and superior parietal regions, which was in line with previous findings (Otten et al., 2012). Typically, a common finding of pathological synchronization in PD is hypersynchronization of the cortical regions in the beta range (Silberstein et al., 2005; George et al., 2013; Pollok et al., 2013). This appears to be related to exaggerated beta synchronization within the BG and between the BG and 


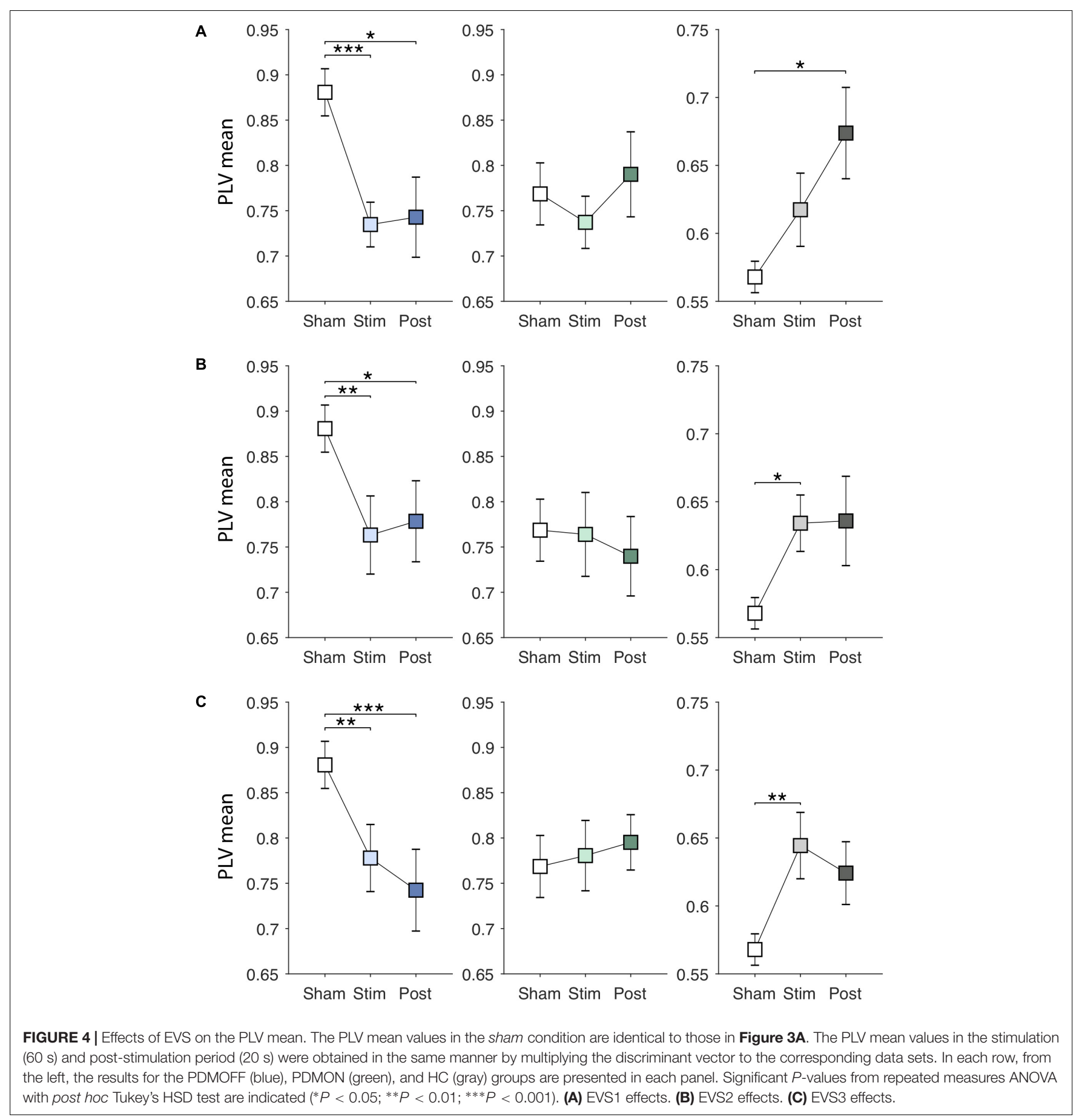

motor cortical regions (Brown, 2007; Jenkinson and Brown, 2011; Brittain and Brown, 2014). However, growing evidence indicates that PD has more complex influences on motor networks beyond excessive beta synchronization (Wu et al., 2009, 2011). There is altered cortical oscillatory activity in other bands beside beta (Bosboom et al., 2006; Stoffers et al., 2007). On the other hand, there is substantial agreement that therapeutic DBS (Pollok et al., 2013) and dopaminergic medication (Wu et al., 2009; HeinrichsGraham et al., 2014; Tahmasian et al., 2015) have normalizing effects on rsFC of motor networks in PD. Consistent with these findings, our results demonstrated that the altered connectivity found in the PDMOFF group was normalized by both medication and EVS to a similar extent.

\section{Variability and Entropy of PLV in the Theta Band}

The altered variability and entropy of PLV in the PD group were mostly found in the theta band (Figures 2B,C), which may reflect abnormalities in thalamocortical dynamics. The 


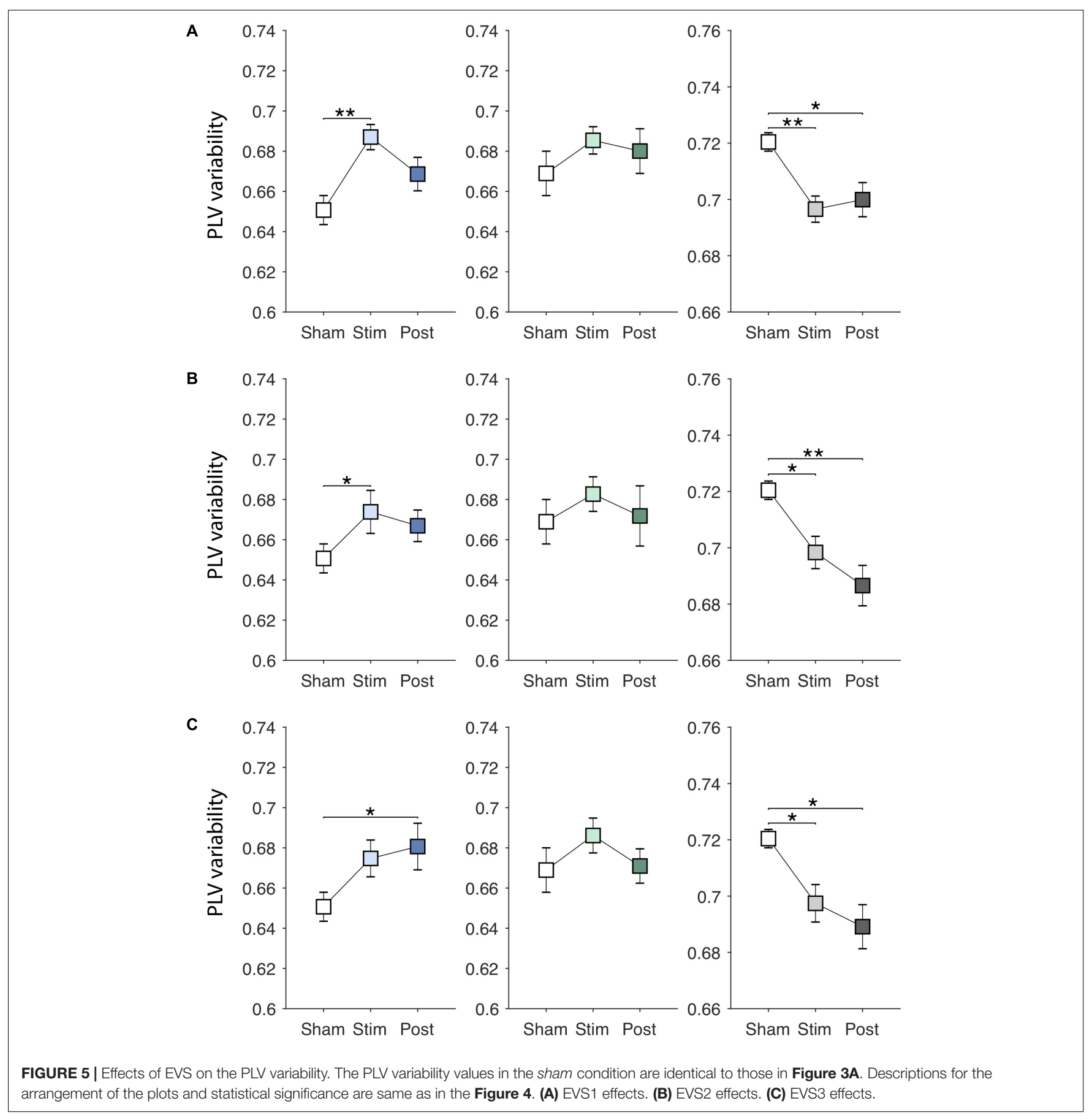

ventral anterior (VA) and anterior part of ventral lateral (VLa) thalamic nuclei are the major recipients from the globus pallidus internus $(\mathrm{GPi})$ via pallidothalamic tracts that are crucially involved in motor disorders such as PD (Gallay et al., 2008). Simultaneously-recorded LFP in the VA and VLa nuclei and EEG on the scalp from PD subjects demonstrated the highest coherence in the theta band (4-9 Hz), in particular in the frontal region of both hemispheres (Sarnthein and Jeanmonod, 2007). Thalamocortical interaction may thus be a major influence in generation of frontal theta activity in $\mathrm{PD}$, and possibly also healthy controls, but we typically do not have LFP recordings from healthy subjects. Multimodal functional imaging studies in healthy human and animal models suggest that the thalamus is critically involved in generating and modulating activities in the cortex (Klimesch, 1999; Schreckenberger et al., 2004; Hughes and Crunelli, 2005; Klimesch et al., 2007). The enhanced synchronization in the theta band of the thalamus and frontal cortical region may be reflective of pathological changes in PD. Together, we conjecture that the increased mean and reduced variability in theta that we observed in PD 


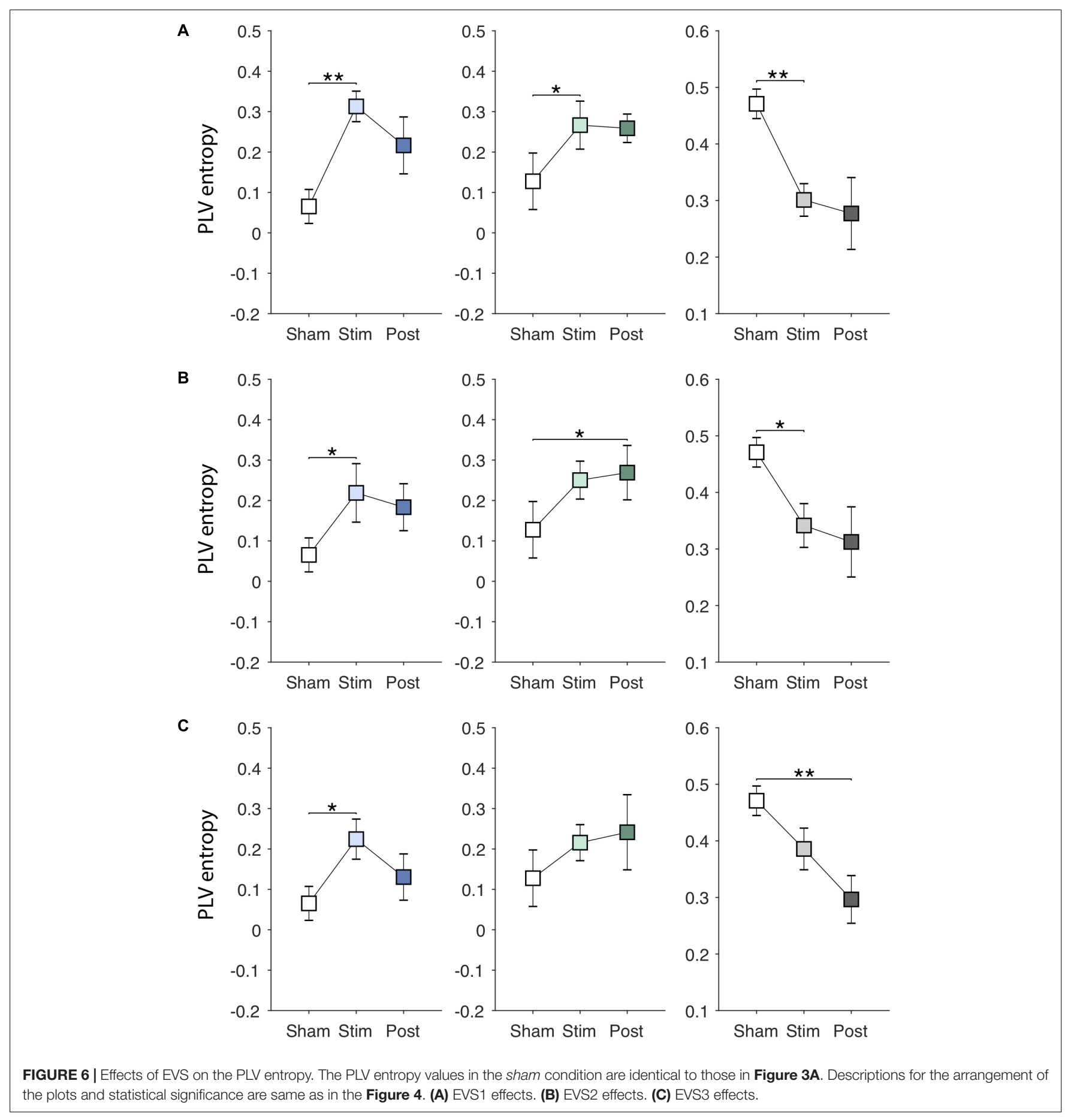

subjects was a consequence of excessive synchronization between thalamocortical structures.

\section{Variability and Entropy of PLV in the Alpha Band}

The dominant frequency in the human EEG under rest is in the alpha frequency band $(8-13 \mathrm{~Hz})$. Alpha oscillations are known to be affected by visual and auditory stimuli (Hari et al., 1997) and change during voluntary movement (Pfurtscheller et al., 2006). A large body of evidence has also demonstrated the critical role of alpha rhythms in attention as well as various cognitive functions (Klimesch, 1999). The dynamic change of alpha activity reflects a variability of states with enhanced and reduced cortical excitability, facilitating the brain's responses to surrounding stimuli (Garrett et al., 2013). Several studies have shown that brain signal variability/complexity can serve as an important discriminator for clinical comparisons. For example, 
EEG entropy is related to brain maturity, as adults have higher entropy compared to children and adolescents (Lippe et al., 2009). Higher entropy is also correlated with better performance on a working memory task (McIntosh et al., 2008). Schlee et al. (2014) found reduced variability of alpha activity during rest over the temporal cortex for subjects with tinnitus compared to controls. Similarly, the reduced variability and complexity of the cortical couplings of the PD groups we observed may be related to diminished motor and cognitive adaptability, as executive cognitive functions such as set shifting, divided or alternating attention and dual tasking (e.g., combining walking with another task) are impaired in PD (Muslimovic et al., 2007; Aarsland et al., 2010; Watson and Leverenz, 2010). Although the mechanisms responsible for these symptoms have not been fully accounted for, dopaminergic depletion in the striatum disrupts the parallel organization of cortico-striatal circuits, resulting in more widespread instead of domain-specific involvement of striatal activity and loss of the normally segregated circuits (Bergman et al., 1998; Calabresi et al., 2015; Nieuwhof et al., 2017). Our results together with the close relationships between cortico-striatal circuits and cortical alpha oscillations (Laufs et al., 2003; Slagter et al., 2017) warrant future studies to further elucidate the functional implications of the impaired alpha dynamic couplings we have demonstrated here.

\section{PLV Sample Entropy Is Higher in the Long-Range Gamma Activity in PD}

We found that the connectivity in the gamma band was more irregular in the PD group than the HC group (Figure 2C). The binding of cortical regions together via synchronization of gamma oscillations between neuronal populations, is implicated in numerous cognitive processes (Fries, 2009; Sohal, 2012). In voluntary movement, for example, synchronization of cortical gamma oscillations prior to movement onset has been described as representing active information processing (Pfurtscheller et al., 1993; Salenius et al., 1996) and considered to serve as a prokinetic signal (Brittain and Brown, 2014). Abnormal gamma oscillations in the motor cortex in $\mathrm{PD}$ have been reported (Litvak et al., 2012; Nowak et al., 2018). However, restingstate gamma oscillations and connectivity in PD remain largely unknown. The mechanism underlying generation of the gamma oscillations are known to be critically involved with excitatory post-synaptic potentials (EPSPs) of gamma-aminobutyric acid (GABA)ergic interneurons and their intact function of fastspiking (Fuchs et al., 2001; Vreugdenhil et al., 2003; Hajos et al., 2004). Thus, alterations in function of GABAergic interneurons could be inferenced from gamma-band oscillations at the macroscopic level. The fast-spiking interneurons are modulated by neurotransmitters including acetylcholine (Fries, 2009; Teles-Grilo Ruivo and Mellor, 2013; Tremblay et al., 2016) and serotonin (Fries, 2009; Puig et al., 2010), and there is robust evidence demonstrating deficits in the cholinergic and serotoninergic systems in PD contributing to various aspects of parkinsonian pathophysiology including motor symptoms, gait dysfunction, cognitive decline, autonomic dysfunction (for review, see Perez-Lloret and Barrantes, 2016). Therefore, it is likely that the disrupted neurotransmitter systems in $\mathrm{PD}$ cause alterations in the activities of fast-spiking interneurons, subsequently resulting in pathological cortical couplings in the gamma band in PD.

\section{Normalizing Effects of EVS and Potential Mechanisms}

In this study, we demonstrated that EVS normalizes the mean, variability and entropy of PLV in PD subjects during stimulation and the extent and duration of the effects were dependent on the stimulation frequencies (Figures 4-6). Modulatory effects of EVS on the cortical oscillatory activity were reported in prior EEG studies that noisy stimulus (pink noise in $0.1-10 \mathrm{~Hz}$ ) decreased gamma oscillatory activity in the lateral regions and increased the beta and gamma activity in the frontal region (Kim et al., 2013), and altered interhemispheric coherence (Lee et al., 2017). To our knowledge, effects of high-frequency multisine EVS $(>50 \mathrm{~Hz})$ on cortical activity have not been explored yet in humans and the results presented in this study provide valuable information on how the effects would differ from low-frequency EVS that has been used in prior behavior and neuroimaging studies. We found two characteristics of effects induced by EVS2 and EVS3 on PLV. First, their effects were similar to EVS1 in the sense that the direction of changes (i.e., increase or decrease in the PLV features) was the same. We did not find a frequency specific increase or decrease in the PLV value in both the PD and HC groups. Second, the extent of changes was less compared to EVS1 during the high-frequency stimulation but lasted longer in the post-stimulation period. This was observed in the PDMOFF group for all the PLV measures and in the HC group for the variability and entropy. For the PDMON group, the EVS effects were less significant, indicating the processing of vestibular inputs in the thalamus and BG (Lopez and Blanke, 2011; Stiles and Smith, 2015; Wijesinghe et al., 2015) is dependent on the dopaminergic level of the BG.

Modulation of firing rates of vestibular afferents by externally applied electrical current will alter directly the vestibular nuclei activities in the brain stem, and eventually multiple cortical areas through the thalamocortical vestibular system. Thus, understanding vestibular information processing regarding varying frequency contents at the vestibular nuclei and thalamus is critical to comprehend above findings. A prior study that examined spiking rates of the guinea pig medial vestibular nuclei (MVN) reported that two types of neurons having different characteristics of afterpotentials responded to current inputs differentially according to the frequency content $(1-30 \mathrm{~Hz}$ ) (Ris et al., 2001). It was shown that spontaneous firing rates of type A neurons was well modulated by only low-frequency $(<10 \mathrm{~Hz})$ current inputs and the spiking rates become irrelevant to the current input at high frequencies whereas type B neurons tended to fire in synchrony better when the stimulation frequency was higher, which demonstrates existence of signal transformation at the vestibular nuclei level to a certain extent in that type A neurons act like a low-pass filter (du Lac and Lisberger, 1995; Ris et al., 2001) whereas type B neurons act as signal detectors with greater sensitivity to external stimuli at high frequencies. 
Considering functional roles of the thalamic nuclei playing integrative and modulatory roles in sensorimotor processing (Tyll et al., 2011), it is likely that further transformation of the modified signal transmitted from the vestibular nuclei occurs in the thalamus. The VA, VL, ventral posterior lateral (VPL), ventral posterior medial (VPM), intraminar nuclei and geniculate bodies of the thalamus receive primary afferents from the vestibular nuclei and play a critical role in processing vestibular information (Bucher et al., 1998; Bense et al., 2001; Stephan et al., 2005; Meng et al., 2007; Wijesinghe et al., 2015). These thalamic nuclei also receive a range of different afferents from peripheral sensory, subcortical, and cortical regions, and process the different types of information before sending the refined signals to the cortex. This may also explain the interaction between EVS and L-dopa medication as observed in the PDMOFF and PDMON groups as the thalamic nuclei processing vestibular information would be receiving differential inputs from the BG according to dopamine levels. Together, unlike the transcranial electrical or magnetic stimulation that directly target cortical regions of interest, influences of EVS on cortical activities are much more indirect. Our results suggest that although the frequency contents of current input to the peripheral vestibular nerve vary considerably, alterations of the refined higher-level multisensory information transmitted from the thalamic nuclei to the broad cortical regions may be relatively consistent.

\section{Limitations}

In the current work, the post-stimulation effects were only evaluated for the first $20 \mathrm{~s}$ after stimulation ceased and there may be potential confounding effects if the after effects persist much longer. Aftereffects of EVS on cortical activation have not been fully investigated yet. Delayed responses in the beta and gamma power in frontal regions was reported to appear 20-25 s after 72-s EVS, but lasted only for several seconds. Based on prior studies reporting aftereffects of invasive (Wingeier et al., 2006) and non-invasive stimulation (Strüber et al., 2015) and the short duration of weak current EVS used here, we concluded that the break time and randomly-ordered trials were sufficient to avoid confounding effects.

\section{CONCLUSION}

In conclusion, in this resting-state EEG study, we demonstrated that connectivity strengths in the sensorimotor region,

\section{REFERENCES}

Aarsland, D., Bronnick, K., Williams-Gray, C., Weintraub, D., Marder, K., Kulisevsky, J., et al. (2010). Mild cognitive impairment in Parkinson disease: a multicenter pooled analysis. Neurology 75, 1062-1069. doi: 10.1212/WNL. 0b013e3181f39d0e

Ahn, S., Zauber, S. E., Worth, R. M., Witt, T., and Rubchinsky, L. L. (2015). Interaction of synchronized dynamics in cortex and basal ganglia in Parkinson's disease. Eur. J. Neurosci. 42, 2164-2171. doi: 10.1111/ejn.12980

Amengual, J. L., Vernet, M., Adam, C., and Valero-Cabré, A. (2017). Local entrainment of oscillatory activity induced by direct brain stimulation in humans. Sci. Rep. 7:41908. doi: 10.1038/srep41908 and variability and complexity of the time-varying corticocortical connectivity are affected in PD, and improved by subthreshold EVS. Furthermore, the magnitude and duration of the improvement was found to vary depending on the stimulation frequency and the subjects' dopamine level. The findings from the current study provide valuable information that thalamic functions of integrating subcortical afferent inputs and thalamocortical projections to the cortex play a critical role in the mechanism of the EVS effects, and warrant further investigation of EVS as a potential therapy in PD.

\section{DATA AVAILABILITY}

The raw data supporting the conclusions of this manuscript will be made available by the authors, without undue reservation, to any qualified researcher.

\section{AUTHOR CONTRIBUTIONS}

SL and MM designed the study. SL conducted the study, performed the data analysis, and wrote the first draft of the manuscript. $\mathrm{AL}$ and $\mathrm{ZW}$ contributed to interpretation of data for the work. All authors contributed to manuscript revision, read, and approved the submitted version.

\section{FUNDING}

This work was partly funded by the Pacific Parkinson's Research Institute (PPRI)/UBC chair in Parkinson's disease (MM) and a generous gift from the Mottershead Foundation. This material was partly based upon work supported by National Natural Science Foundation of China (61701158).

\section{ACKNOWLEDGMENTS}

We would like to thank Christy Jones for assisting recruiting participants, and are enormously grateful to the participants in this study for generous support.
Andres, F. G., and Gerloff, C. (1999). Coherence of sequential movements and motor learning. J. Clin. Neurophysiol. 16, 520-527. doi: 10.1097/00004691199911000-00004

Bense, S., Stephan, T., Yousry, T. A., Brandt, T., and Dieterich, M. (2001). Multisensory cortical signal increases and decreases during vestibular galvanic stimulation (fMRI). J. Neurophysiol. 85, 886-899. doi: 10.1152/jn.2001.85.2.886

Bergman, H., Feingold, A., Nini, A., Raz, A., Slovin, H., Abeles, M., et al. (1998). Physiological aspects of information processing in the basal ganglia of normal and parkinsonian primates. Trends Neurosci. 21, 32-38. doi: 10.1016/S01662236(97)01151-X

Bosboom, J. L., Stoffers, D., Stam, C. J. J., van Dijk, B. W. W., Verbunt, J., Berendse, H. W. W., et al. (2006). Resting state oscillatory brain dynamics in Parkinson's 
disease: an MEG study. Clin. Neurophysiol. 117, 2521-2531. doi: 10.1016/j. clinph.2006.06.720

Brittain, J.-S., and Brown, P. (2014). Oscillations and the basal ganglia: motor control and beyond. Neuroimage 85(Pt 2), 637-647. doi: 10.1016/j.neuroimage. 2013.05.084

Brown, P. (2007). Abnormal oscillatory synchronisation in the motor system leads to impaired movement. Curr. Opin. Neurobiol. 17, 656-664. doi: 10.1016/j.conb. 2007.12.001

Brown, P., Oliviero, A., Mazzone, P., Insola, A., Tonali, P., and Di Lazzaro, V. (2001). Dopamine dependency of oscillations between subthalamic nucleus and pallidum in Parkinson's disease. J. Neurosci. 21, 1033-1038. doi: 10.1523/ JNEUROSCI.21-03-01033.2001

Brown, P., and Williams, D. (2005). Basal ganglia local field potential activity: character and functional significance in the human. Clin. Neurophysiol. 116, 2510-2519. doi: 10.1016/j.clinph.2005.05.009

Bruce, E. N., Bruce, M. C., and Vennelaganti, S. (2009). Sample entropy tracks changes in electroencephalogram power spectrum with sleep state and aging. J. Clin. Neurophysiol. 26, 257-266. doi: 10.1097/WNP.0b013e3181b2f1e3

Bruña, R., Maestú, F., and Pereda, E. (2018). Phase locking value revisited: teaching new tricks to an old dog. J. Neural Eng. 15:056011. doi: 10.1088/1741-2552/ aacfe4

Bucher, S. F., Dieterich, M., Wiesmann, M., Weiss, A., Zink, R., Yousry, T. A., et al. (1998). Cerebral functional magnetic resonance imaging of vestibular, auditory, and nociceptive areas during galvanic stimulation. Ann. Neurol 44, 120-125. doi: $10.1002 /$ ana.410440118

Calabresi, P., Ghiglieri, V., Mazzocchetti, P., Corbelli, I., and Picconi, B. (2015). Levodopa-induced plasticity: a double-edged sword in Parkinson's disease? Philos. Trans. R. Soc. B Biol. Sci. 370:20140184. doi: 10.1098/rstb.2014.0184

Cassidy, M., Mazzone, P., Oliviero, A., Insola, A., Tonali, P., Di Lazzaro, V., et al. (2002). Movement-related changes in synchronization in the human basal ganglia. Brain 125, 1235-1246. doi: 10.1093/brain/awf135

Clemmensen, L., Hastie, T., Witten, D., and Ersbøll, B. (2011). Sparse Discriminant Analysis. Technometrics 53, 406-413. doi: 10.1198/TECH.2011.08118

Davie, C. A. (2008). A review of Parkinson's disease. Br. Med. Bull. 86, 109-127. doi: $10.1093 / \mathrm{bmb} / \mathrm{ldn} 013$

du Lac, S., and Lisberger, S. G. (1995). Cellular processing of temporal information in medial vestibular nucleus neurons. J. Neurosci. 15, 8000-8010. doi: 10.1523/ JNEUROSCI.15-12-08000.1995

Dubbelink, K. T. O., Schoonheim, M. M., Deijen, J. B., Twisk, J. W. R., Barkhof, F., Berendse, H. W., et al. (2014). Functional connectivity and cognitive decline over 3 years in Parkinson disease. Neurology 83, 2046-2053. doi: 10.1212/WNL. 0000000000001020

Eusebio, A., Pogosyan, A., Wang, S., Averbeck, B., Gaynor, L. D., Cantiniaux, S., et al. (2009). Resonance in subthalamo-cortical circuits in Parkinson's disease. Brain 132, 2139-2150. doi: 10.1093/brain/awp079

Fell, J., and Axmacher, N. (2011). The role of phase synchronization in memory processes. Nat. Rev. Neurosci. 12, 105-118. doi: 10.1038/nrn2979

Fries, P. (2009). Neuronal gamma-band synchronization as a fundamental process in cortical computation. Annu. Rev. Neurosci. 32, 209-224. doi: 10.1146/ annurev.neuro.051508.135603

Fuchs, E. C., Doheny, H., Faulkner, H., Caputi, A., Traub, R. D., Bibbig, A., et al. (2001). Genetically altered AMPA-type glutamate receptor kinetics in interneurons disrupt long-range synchrony of gamma oscillation. Proc. Natl. Acad. Sci. U.S.A. 98, 3571-3576. doi: 10.1073/pnas.05163 1898

Gallay, M. N., Jeanmonod, D., Liu, J., and Morel, A. (2008). Human pallidothalamic and cerebellothalamic tracts: anatomical basis for functional stereotactic neurosurgery. Brain Struct. Funct. 212, 443-463. doi: 10.1007/s00429-0070170-0

Garrett, D. D., Samanez-Larkin, G. R., MacDonald, S. W. S., Lindenberger, U., McIntosh, A. R., and Grady, C. L. (2013). Moment-to-moment brain signal variability: a next frontier in human brain mapping? Neurosci. Biobehav. Rev. 37, 610-624. doi: 10.1016/j.neubiorev.2013.02.015

George, J. S., Strunk, J., Mak-McCully, R., Houser, M., Poizner, H., and Aron, A. R. (2013). Dopaminergic therapy in Parkinson's disease decreases cortical beta band coherence in the resting state and increases cortical beta band power during executive control. Neuroimage Clin. 3, 261-270. doi: 10.1016/j.nicl.2013. 07.013
Groppe, D. M., Bickel, S., Keller, C. J., Jain, S. K., Hwang, S. T., Harden, C., et al. (2013). Dominant frequencies of resting human brain activity as measured by the electrocorticogram. Neuroimage 79, 223-233. doi: 10.1016/j.neuroimage. 2013.04.044

Hajos, N., Pálhalmi, J., Mann, E. O., Németh, B., Paulsen, O., and Freund, T. F. (2004). Spike timing of distinct types of GABAergic interneuron during hippocampal gamma oscillations in vitro. J. Neurosci. 24, 9127-9137. doi: 10.1523/JNEUROSCI.2113-04.2004

Hanslmayr, S., Klimesch, W., Sauseng, P., Gruber, W., Doppelmayr, M., Freunberger, R., et al. (2005). Visual discrimination performance is related to decreased alpha amplitude but increased phase locking. Neurosci. Lett. 375, 64-68. doi: 10.1016/j.neulet.2004.10.092

Hari, R., Salmelin, R., Mäkelä, J. P., Salenius, S., and Helle, M. (1997). Magnetoencephalographic cortical rhythms. Int. J. Psychophysiol. 26, 51-62. doi: 10.1016/S0167-8760(97)00755-1

He, X., Zhang, Y., Chen, J., Xie, C., Gan, R., Yang, R., et al. (2017). The patterns of EEG changes in early-onset Parkinson's disease patients. Int. J. Neurosci. 127, 1028-1035. doi: 10.1080/00207454.2017.1304393

Heinrichs-Graham, E., Kurz, M. J., Becker, K. M., Santamaria, P. M., Gendelman, H. E., and Wilson, T. W. (2014). Hypersynchrony despite pathologically reduced beta oscillations in patients with Parkinson's disease: a pharmacomagnetoencephalography study. J. Neurophysiol. 112, 1739-1747. doi: 10.1152/ jn.00383.2014

Helfrich, R. F. F., Schneider, T. R. R., Rach, S., Trautmann-Lengsfeld, S. A. A., Engel, A. K. K., and Herrmann, C. S. S. (2014). Entrainment of brain oscillations by transcranial alternating current stimulation. Curr. Biol. 24, 333-339. doi: 10.1016/j.cub.2013.12.041

Helmich, R. C., Derikx, L. C., Bakker, M., Scheeringa, R., Bloem, B. R., and Toni, I. (2010). Spatial remapping of cortico-striatal connectivity in Parkinson's disease. Cereb. Cortex 20, 1175-1186. doi: 10.1093/cercor/bhp178

Hughes, S. W., and Crunelli, V. (2005). Thalamic mechanisms of EEG alpha rhythms and their pathological implications. Neuroscientist 11, 357-372. doi: $10.1177 / 1073858405277450$

Jenkinson, N., and Brown, P. (2011). New insights into the relationship between dopamine, beta oscillations and motor function. Trends Neurosci. 34, 611-618. doi: 10.1016/j.tins.2011.09.003

Kim, D. J., Yogendrakumar, V., Chiang, J., Ty, E., Wang, Z. J., and McKeown, M. J. (2013). Noisy galvanic vestibular stimulation modulates the amplitude of EEG synchrony patterns. PLoS One 8:e69055. doi: 10.1371/journal.pone.0069055

Klimesch, W. (1999). EEG alpha and theta oscillations reflect cognitive and memory performance: a review and analysis. Brain Res. Rev. 29, 169-195. doi: 10.1016/S0165-0173(98)00056-3

Klimesch, W., Doppelmayr, M., Yonelinas, A., Kroll, N. E., Lazzara, M., Röhm, D., et al. (2001). Theta synchronization during episodic retrieval: neural correlates of conscious awareness. Brain Res. Cogn. Brain Res. 12, 33-38. doi: 10.1016/ S0926-6410(01)00024-6

Klimesch, W., Freunberger, R., Sauseng, P., and Gruber, W. (2008). A short review of slow phase synchronization and memory: evidence for control processes in different memory systems? Brain Res. 1235, 31-44. doi: 10.1016/J.BRAINRES. 2008.06.049

Klimesch, W., Sauseng, P., and Hanslmayr, S. (2007). EEG alpha oscillations: the inhibition-timing hypothesis. Brain Res. Rev. 53, 63-88. doi: 10.1016/j. brainresrev.2006.06.003

Kumar, Y., Dewal, M. L., and Anand, R. S. (2012). "Features extraction of EEG signals using approximate and sample entropy," in Proceedings of the 2012 IEEE Students' Conference on Electrical, Electronics and Computer Science (IEEE); 2012 March 1-2, Bhopal, 1-5. doi: 10.1109/SCEECS.2012. 6184830

Lachaux, J. P., Rodriguez, E., Martinerie, J., and Varela, F. J. (1999). Measuring phase synchrony in brain signals. Hum. Brain Mapp. 8, 194-208. doi: 10.1002/(SICI) 1097-0193(1999)8:4<194::AID-HBM4>3. $0 . \mathrm{CO} ; 2-\mathrm{C}$

Lake, D. E., Richman, J. S., Griffin, M. P., and Moorman, J. R. (2002). Sample entropy analysis of neonatal heart rate variability. Am. J. Physiol. Integr. Comp. Physiol. 283, R789-R797. doi: 10.1152/ajpregu.00069.2002

Laufs, H., Kleinschmidt, A., Beyerle, A., Eger, E., Salek-Haddadi, A., Preibisch, C., et al. (2003). EEG-correlated fMRI of human alpha activity. Neuroimage 19, 1463-1476. doi: 10.1016/S1053-8119(03)00286-6 
Lee, S., Kim, D., and McKeown, M. J. (2017). "Galvanic Vestibular Stimulation (GVS) effects on impaired interhemispheric connectivity in Parkinson's Disease," in Proceedings of the 2017 39th Annual International Conference of the IEEE Engineering in Medicine and Biology Society (IEEE); 2017 July 11-15, Jeju, 2109-2113. doi: 10.1109/EMBC.2017.8037270

Lee, S., Kim, D. J., Svenkeson, D., Parras, G., Oishi, M. M. K., and McKeown, M. J. (2015). Multifaceted effects of noisy galvanic vestibular stimulation on manual tracking behavior in Parkinson's disease. Front. Syst. Neurosci. 9:5. doi: 10.3389/fnsys.2015.00005

Lee, S., McKeown, M. J., Wang, Z. J., and Chen, X. (2019). Removal of highvoltage brain stimulation artifacts from simultaneous EEG recordings. IEEE Trans. Biomed. Eng. 66, 50-60. doi: 10.1109/TBME.2018.2828808

Lippe, S., Kovacevic, N., and McIntosh, A. R. (2009). Differential maturation of brain signal complexity in the human auditory and visual system. Front. Hum. Neurosci. 3:48. doi: 10.3389/neuro.09.048.2009

Litvak, V., Eusebio, A., Jha, A., Oostenveld, R., Barnes, G., Foltynie, T., et al. (2012). Movement-related changes in local and long-range synchronization in Parkinson's disease revealed by simultaneous magnetoencephalography and intracranial recordings. J. Neurosci. 32, 10541-10553. doi: 10.1523/ JNEUROSCI.0767-12.2012

Lopez, C., and Blanke, O. (2011). The thalamocortical vestibular system in animals and humans. Brain Res. Rev. 67, 119-146. doi: 10.1016/j.brainresrev.2010. 12.002

Lopez, C., Blanke, O., and Mast, F. W. (2012). The human vestibular cortex revealed by coordinate-based activation likelihood estimation meta-analysis. Neuroscience 212, 159-179. doi: 10.1016/j.neuroscience.2012.03.028

Mai, Q. (2013). A review of discriminant analysis in high dimensions. Wiley Interdiscip. Rev. Comput. Stat. 5, 190-197. doi: 10.1002/wics.1257

Marsden, J. F., Limousin-Dowsey, P., Ashby, P., Pollak, P., and Brown, P. (2001). Subthalamic nucleus, sensorimotor cortex and muscle interrelationships in Parkinson's disease. Brain 124, 378-388. doi: 10.1093/brain/124.2.378

Martin, K. A. C., and Schröder, S. (2016). Phase locking of multiple single neurons to the local field potential in cat V1. J. Neurosci. 36, 2494-2502. doi: 10.1523/ JNEUROSCI.2547-14.2016

McIntosh, A. R., Kovacevic, N., and Itier, R. J. (2008). Increased brain signal variability accompanies lower behavioral variability in development. PLoS Comput. Biol. 4:e1000106. doi: 10.1371/journal.pcbi.1000106

Meng, H., May, P. J., Dickman, J. D., and Angelaki, D. E. (2007). Vestibular signals in primate thalamus: properties and origins. J. Neurosci. 27, 13590-13602. doi: 10.1523/JNEUROSCI.3931-07.2007

Moazami-Goudarzi, M., Sarnthein, J., Michels, L., Moukhtieva, R., and Jeanmonod, D. (2008). Enhanced frontal low and high frequency power and synchronization in the resting EEG of Parkinsonian patients. Neuroimage 41, 985-997. doi: 10.1016/j.neuroimage.2008.03.032

Mormann, F., Lehnertz, K., David, P., and Elger, C. (2000). Mean phase coherence as a measure for phase synchronization and its application to the EEG of epilepsy patients. Phys. D Nonlinear Phenom. 144, 358-369. doi: 10.1016/S01672789(00)00087-7

Muslimovic, D., Schmand, B., Speelman, J. D., and De Haan, R. J. (2007). Course of cognitive decline in Parkinson's disease: a meta-analysis. J. Int. Neuropsychol. Soc. 13, 920-932. doi: 10.1017/S1355617707071160

Nieuwhof, F., Bloem, B. R., Reelick, M. F., Aarts, E., Maidan, I., Mirelman, A., et al. (2017). Impaired dual tasking in Parkinson's disease is associated with reduced focusing of cortico-striatal activity. Brain 140, 1384-1398. doi: 10.1093/brain/ awx042

Nowak, M., Zich, C., and Stagg, C. J. (2018). Motor cortical gamma oscillations: What have we learnt and where are we headed?. Curr. Behav. Neurosci. Rep. 5, 136-142. doi: 10.1007/s40473-018-0151-z

Oswal, A., Brown, P., and Litvak, V. (2013). Synchronized neural oscillations and the pathophysiology of Parkinson's disease. Curr. Opin. Neurol. 26, 662-670. doi: 10.1097/WCO.0000000000000034

Otten, M. L., Mikell, C. B., Youngerman, B. E., Liston, C., Sisti, M. B., Bruce, J. N., et al. (2012). Motor deficits correlate with resting state motor network connectivity in patients with brain tumours. Brain 135, 1017-1026. doi: 10. 1093/brain/aws041

Pan, W., Soma, R., Kwak, S., and Yamamoto, Y. (2008). Improvement of motor functions by noisy vestibular stimulation in central neurodegenerative disorders. J. Neurol. 255, 1657-1661. doi: 10.1007/s00415-008-0950-3
Perez-Lloret, S., and Barrantes, F. J. (2016). Deficits in cholinergic neurotransmission and their clinical correlates in Parkinson's disease. NPJ Park. Dis. 2:16001. doi: 10.1038/npjparkd.2016.1

Pfurtscheller, G., Brunner, C., Schlögl, A., and Lopes da Silva, F. H. (2006). Mu rhythm (de)synchronization and EEG single-trial classification of different motor imagery tasks. Neuroimage 31, 153-159. doi: 10.1016/J.NEUROIMAGE. 2005.12.003

Pfurtscheller, G., Neuper, C., and Kalcher, J. (1993). 40-Hz oscillations during motor behavior in man. Neurosci. Lett. 164, 179-182. doi: 10.1016/03043940(93)90886-P

Pollok, B., Kamp, D., Butz, M., Wojtecki, L., Timmermann, L., Südmeyer, M., et al. (2013). Increased SMA-M1 coherence in Parkinson's disease Pathophysiology or compensation? Exp. Neurol. 247, 178-181. doi: 10.1016/j. expneurol.2013.04.013

Puig, M. V., Watakabe, A., Ushimaru, M., Yamamori, T., and Kawaguchi, Y. (2010). Serotonin modulates fast-spiking interneuron and synchronous activity in the rat prefrontal cortex through 5-HT1A and 5-HT2A receptors. J. Neurosci. 30, 2211-2222. doi: 10.1523/JNEUROSCI.3335-09.2010

Richman, J. S., and Moorman, J. R. (2000). Physiological time-series analysis using approximate entropy and sample entropy. Am. J. Physiol. Circ. Physiol. 278, H2039-H2049. doi: 10.1152/ajpheart.2000.278.6.H2039

Ris, L., Hachemaoui, M., Vibert, N., Godaux, E., Vidal, P. P., and Moore, L. E. (2001). Resonance of spike discharge modulation in neurons of the guinea pig medial vestibular nucleus. J. Neurophysiol. 86, 703-716. doi: 10.1152/jn.2001. 86.2.703

Safo, S. E., and Long, Q. (2018). Sparse linear discriminant analysis in structured covariates space. Stat. Anal. Data Min. 2018, 1-14. doi: 10.1002/sam.11376

Sakkalis, V., Tsiaras, V., Zervakis, M., and Tollis, I. (2007). "Optimal brain network synchrony visualization: application in an alcoholism paradigm," in Proceedings of the 2007 29th Annual International Conference of the IEEE Engineering in Medicine and Biology Society (IEEE); 2007 August 22-26, Lyon, 4285-4288. doi: 10.1109/IEMBS.2007.4353283

Salenius, S., Salmelin, R., Neuper, C., Pfurtscheller, G., and Hari, R. (1996). Human cortical $40 \mathrm{~Hz}$ rhythm is closely related to EMG rhythmicity. Neurosci. Lett. 213, 75-78. doi: 10.1016/0304-3940(96)12796-8

Sarnthein, J., and Jeanmonod, D. (2007). High thalamocortical theta coherence in patients with Parkinson's disease. J. Neurosci. 27, 124-131. doi: 10.1523/ JNEUROSCI.2411-06.2007

Sauseng, P., and Klimesch, W. (2008). What does phase information of oscillatory brain activity tell us about cognitive processes? Neurosci. Biobehav. Rev. 32, 1001-1013. doi: 10.1016/j.neubiorev.2008.03.014

Scandalis, T. A., Bosak, A., Berliner, J. C., Helman, L. L., and Wells, M. R. (2001). Resistance training and gait function in patients with Parkinson's disease. Am. J. Phys. Med. Rehabil. 80, 38-43. doi: 10.1097/00002060-200101000-00011

Schlee, W., Schecklmann, M., Lehner, A., Kreuzer, P. M., Vielsmeier, V., Poeppl, T. B., et al. (2014). Reduced variability of auditory alpha activity in chronic tinnitus. Neural Plast. 2014:436146. doi: 10.1155/2014/436146

Schreckenberger, M., Lange-Asschenfeldt, C., Lange-Asschenfeld, C., Lochmann, M., Mann, K., Siessmeier, T., et al. (2004). The thalamus as the generator and modulator of EEG alpha rhythm: a combined PET/EEG study with lorazepam challenge in humans. Neuroimage 22, 637-644. doi: 10.1016/j.neuroimage.2004.01.047

Seibert, T. M., Murphy, E. A., Kaestner, E. J., and Brewer, J. B. (2012). Interregional correlations in parkinson disease and parkinson-related dementia with resting functional MR imaging. Radiology 263, 226-234. doi: 10.1148/radiol.12111280

Silberstein, P., Pogosyan, A., Kühn, A. A., Hotton, G., Tisch, S., Kupsch, A., et al. (2005). Cortico-cortical coupling in Parkinson's disease and its modulation by therapy. Brain 128, 1277-1291. doi: 10.1093/brain/awh480

Slagter, H. A., Mazaheri, A., Reteig, L. C., Smolders, R., Figee, M., Mantione, M., et al. (2017). Contributions of the ventral striatum to conscious perception: an intracranial eeg study of the attentional blink. J. Neurosci. 37, 1081-1089. doi: 10.1523/JNEUROSCI.2282-16.2016

Smolders, R., Mazaheri, A., van Wingen, G., Figee, M., de Koning, P. P., and Denys, D. (2013). Deep brain stimulation targeted at the nucleus accumbens decreases the potential for pathologic network communication. Biol. Psychiatry 74, e27-e28. doi: 10.1016/j.biopsych.2013.03.012

Sohal, V. S. (2012). Insights into cortical oscillations arising from optogenetic studies. Biol. Psychiatry 71, 1039-1045. doi: 10.1016/j.biopsych.2012.01.024 
Song, Y., Crowcroft, J., and Zhang, J. (2012). Automatic epileptic seizure detection in EEGs based on optimized sample entropy and extreme learning machine. J. Neurosci. Methods 210, 132-146. doi: 10.1016/J.JNEUMETH.2012.07.003

Spencer, K. M., Nestor, P. G., Perlmutter, R., Niznikiewicz, M. A., Klump, M. C., Frumin, M., et al. (2004). Neural synchrony indexes disordered perception and cognition in schizophrenia. Proc. Natl. Acad. Sci. U.S.A. 101, 17288-17293. doi: 10.1073/pnas.0406074101

St George, R. J., and Fitzpatrick, R. C. (2011). The sense of self-motion, orientation and balance explored by vestibular stimulation. J. Physiol. 589, 807-813. doi: 10.1113/jphysiol.2010.197665

Stephan, T., Deutschländer, A., Nolte, A., Schneider, E., Wiesmann, M., Brandt, T., et al. (2005). Functional MRI of galvanic vestibular stimulation with alternating currents at different frequencies. Neuroimage 26, 721-732. doi: 10.1016/j. neuroimage.2005.02.049

Stiles, L., and Smith, P. F. (2015). The vestibular-basal ganglia connection: balancing motor control. Brain Res. 1597, 180-188. doi: 10.1016/J.BRAINRES. 2014.11.063

Stoffers, D., Bosboom, J. L. W., Deijen, J. B., Wolters, E. C., Berendse, H. W., and Stam, C. J. (2007). Slowing of oscillatory brain activity is a stable characteristic of Parkinson's disease without dementia. Brain 130, 1847-1860. doi: 10.1093/ brain/awm034

Strüber, D., Rach, S., Neuling, T., and Herrmann, C. S. (2015). On the possible role of stimulation duration for after-effects of transcranial alternating current stimulation. Front. Cell. Neurosci. 9:311. doi: 10.3389/fncel.2015.00311

Tahmasian, M., Bettray, L. M., van Eimeren, T., Drzezga, A., Timmermann, L., Eickhoff, C. R., et al. (2015). A systematic review on the applications of restingstate fMRI in Parkinson's disease: Does dopamine replacement therapy play a role? Cortex 73, 80-105. doi: 10.1016/j.cortex.2015.08.005

Teles-Grilo Ruivo, L. M., and Mellor, J. R. (2013). Cholinergic modulation of hippocampal network function. Front. Synaptic Neurosci. 5:2. doi: 10.3389/ fnsyn.2013.00002

Tomlinson, C. L., Stowe, R., Patel, S., Rick, C., Gray, R., and Clarke, C. E. (2010). Systematic review of levodopa dose equivalency reporting in Parkinson's disease. Mov. Disord. 25, 2649-2653. doi: 10.1002/mds.23429

Tremblay, R., Lee, S., and Rudy, B. (2016). GABAergic interneurons in the neocortex: from cellular properties to circuits. Neuron 91, 260-292. doi: 10.1016/j.neuron.2016.06.033

Tyll, S., Budinger, E., and Noesselt, T. (2011). Thalamic influences on multisensory integration. Commun. Integr. Biol. 4, 378-381. doi: 10.4161/cib.4.4.15222

Utz, K. S., Dimova, V., Oppenländer, K., and Kerkhoff, G. (2010). Electrified minds: Transcranial direct current stimulation (tDCS) and Galvanic Vestibular Stimulation (GVS) as methods of non-invasive brain stimulation in neuropsychology-A review of current data and future implications. Neuropsychologia 48, 2789-2810. doi: 10.1016/j.neuropsychologia.2010.06.002

Vakorin, V. A., Doesburg, S. M., da Costa, L., Jetly, R., Pang, E. W., and Taylor, M. J. (2016). Detecting mild traumatic brain injury using resting state magnetoencephalographic connectivity. PLoS Comput. Biol. 12:e1004914 doi: 10.1371/JOURNAL.PCBI.1004914

Van der Ouderaa, E., Schoukens, J., and Renneboog, J. (1988). Peak factor minimization of input and output signals of linear systems. IEEE Trans. Instrum. Meas. 37, 207-212. doi: 10.1109/19.6053

Vossen, A., Gross, J., and Thut, G. (2015). Alpha power increase after transcranial alternating current stimulation at alpha frequency ( $\alpha$-tACS) reflects plastic changes rather than entrainment. Brain Stimul. 8, 499-508. doi: 10.1016/j.brs. 2014.12.004

Vreugdenhil, M., Jefferys, J. G. R., Celio, M. R., and Schwaller, B. (2003). Parvalbumin-deficiency facilitates repetitive IPSCs and gamma oscillations in the hippocampus. J. Neurophysiol. 89, 1414-1422. doi: 10.1152/jn.00576.2002

Watson, G. S., and Leverenz, J. B. (2010). Profile of cognitive impairment in Parkinson's disease. Brain Pathol. 20, 640-645. doi: 10.1111/j.1750-3639.2010. 00373.x

Wijesinghe, R., Protti, D. A., and Camp, A. J. (2015). Vestibular interactions in the thalamus. Front. Neural Circuits 9:79. doi: 10.3389/fncir.2015.00079

Williams, D., Tijssen, M., Van Bruggen, G., Bosch, A., Insola, A., Di Lazzaro, V., et al. (2002). Dopamine-dependent changes in the functional connectivity between basal ganglia and cerebral cortex in humans. Brain 125, 1558-1569. doi: 10.1093/brain/awf156

Wingeier, B., Tcheng, T., Koop, M. M., Hill, B. C., Heit, G., and Bronte-Stewart, H. M. (2006). Intra-operative STN DBS attenuates the prominent beta rhythm in the STN in Parkinson's disease. Exp. Neurol. 197, 244-251. doi: 10.1016/j. expneurol.2005.09.016

Wu, T., Long, X., Wang, L., Hallett, M., Zang, Y., Li, K., et al. (2011). Functional connectivity of cortical motor areas in the resting state in Parkinson's disease. Hum. Brain Mapp. 32, 1443-1457. doi: 10.1002/hbm.21118

Wu, T., Wang, L., Chen, Y., Zhao, C., Li, K., and Chan, P. (2009). Changes of functional connectivity of the motor network in the resting state in Parkinson's disease. Neurosci. Lett. 460, 6-10. doi: 10.1016/j.neulet.2009.05.046

Yamamoto, Y., Struzik, Z. R., Soma, R., Ohashi, K., and Kwak, S. (2005). Noisy vestibular stimulation improves autonomic and motor responsiveness in central neurodegenerative disorders. Ann. Neurol. 58, 175-181. doi: 10.1002/ana. 20574

Conflict of Interest Statement: The authors declare that the research was conducted in the absence of any commercial or financial relationships that could be construed as a potential conflict of interest.

Copyright (c) 2019 Lee, Liu, Wang and McKeown. This is an open-access article distributed under the terms of the Creative Commons Attribution License (CC BY). The use, distribution or reproduction in other forums is permitted, provided the original author(s) and the copyright owner(s) are credited and that the original publication in this journal is cited, in accordance with accepted academic practice. No use, distribution or reproduction is permitted which does not comply with these terms. 